GANIT J. Bangladesh Math. Soc. (ISSN 1606-3694) 37 (2017) 99-109

\title{
HAWKING NON-THERMAL AND PURELY THERMAL RADIATIONS OF ROTATING BLACK HOLE IN ANTI-DE SITTER SPACE TIME BY HAMILTON-JACOBI METHOD
}

\author{
M. Ilias Hossain \\ Department of Mathematics, Rajshahi University, Rajshahi-6205, Bangladesh \\ E-mail: ilias_math@yahoo.com
}

Received 16.05.2017 Accepted 17.08.2017

\begin{abstract}
We have explored Hawking non-thermal and purely thermal radiations of Kerr-anti-de Sitter (KAdS) black hole using massive particles tunneling method by taking into account the space time background as dynamical, energy and angular momentum as conserved incorporating the selfgravitation effect of the emitted particles. The results we have obtained for KAdS black hole have shown that the tunneling rates are related to the change of Bekenstein-Hawking entropy and the derived emission spectrum deviates from the pure thermal spectrum and also the obtaining results for KAdS black hole are accordant with Parikh and Wilczek's opinion and gives a correction to the Hawking radiation of KAdS black hole.
\end{abstract}

Keywords: Massive Particle Tunneling, KAdS black hole, Non-thermal and purely thermal radiations.

\section{Introduction}

About four decades ago, an extraordinary invention made by Stephen William Hawking that black holes radiate thermally. By the black hole thermodynamics, the thermal radiation with the Hawking temperature determined by the surface gravity at the event horizon $[12,13]$ is taken as entropy [4-6] and surface gravity is the acceleration measured at the spatial infinity that a stationary particle should undergo to withstand the gravity at the event horizon. The one is the information lost and the other one is the technical problem arisen during the study of Hawking thermal radiation. The loss of information was not a serious problem in the classical theory, since the information could be thought of as preserved inside the black hole but just not very accessible. However, taking the quantum effect into consideration, the situation is changed. On the thermal radiation of black holes [14, 19, 20, 36], the emission of Hawking radiation [12, 13], black holes could lose energy, shrink, and eventually becomes smaller and smaller until disappears completely. It seems that pure quantum states can evolve into mixed states and such an evolution violates the fundamental principles of quantum theory, as these prescribe a unitary time evolution of basis states. Derivations in string theory support the idea that Hawking radiation can be described within a manifestly unitary theory, it remains a mystery how information is returned. Hawking described 
the existence of black hole radiation as tunneling triggered by vacuum fluctuations near the horizon $[12,13]$ but actual derivation of Hawking radiation did not proceed in this way at all [13]. This method also gives a leading correction to the emission rate arising from loss of mass of the black hole corresponding to the energy carried by the radiated quantum. Carrying this method, the Hawking radiation from AdS black holes have investigated by Hemming and Keski-Vakkuri [14] and Medved has studied those from a de Sitter cosmological horizon [28]. All these spherically symmetric investigations are successful. Many researchers developed two universal methods to correctly recover Hawking radiation of black holes. First one is the gravitational anomaly method [39] and other is the semi-classical tunneling method initiated by Kraus and Wilczek [19, 20]. Actually their development is not advanced since that the method applied, is limited to discuss the tunneling rate of the uncharged massless particles only $[1,2,8,9,16,21,22,25,26,29-32,34,37$, $43,48-55]$. For black holes with a charge, the emitted outgoing particles can be charged also, not only should the energy conservation but also the charge conservation be considered $[15,20]$.

Parikh-Wilczek's [36] original calculation only considered the tunneling process of a massless and uncharged particle, recently it is shown that such tunneling method can be easily extended to study the massive [54] and charged particle's tunneling process [25]. Zhang and Zhao was first proposed by Hawking radiation from massive uncharged particle tunneling [57] and charged particle tunneling [58] from black hole and in 2005, Zhang and Zhao et al. extended their work to the Hawking radiation of massive and charged particles and made a great deal of success [17, 55-57, 59], which has effective significance on the further cognition and research on black holes and also in the same year, a different method was introduced by Angheben et al. [3]. It is called HamiltonJacobi method and it is an extension of the complex path analysis proposed by Padmanabhan et al. [44-46]. Using the null-geodesic and Hamilton-Jacobi methods, for analyzing the temperature of Taub-NUT black holes [23], Kerner and Mann [23] developed quantum tunneling methods and Hamilton-Jacobi method is rolled up for calculating the relativistic Hamilton-Jacobi equation. Parikh and Wilczek applied the semi-classical quantum tunneling model to research on the Hawking radiation of the static Schwarzschild and Reissner-Nordstrm black holes [2, 31] and them opinion the true radiation spectrum is not strictly thermal but satisfies the underlying unitary theory when self-gravitation interaction and energy conservation are considered. It is clear that the background geometry of a radiating black hole should be altered (unfixed) with the loss of energy. Chen, $\mathrm{Zu}$ and Yang reformed Hamilton-Jacobi method for massive particle tunneling and investigate the Hawking radiation of the Taub-NUT black hole [10] and using this method Hawking radiation of Kerr-NUT black hole [27] and the charged black hole with a global monopole [18] developed using Painlev'e coordinate system. In this paper, this method is applied to investigate the Hawking radiation of KAdS black hole and to calculate the imaginary part of action from Hamilton-Jacobi equation avoid by exploring the equation of motion of the radiation particle in Painlev'e coordinate system and calculating the Hamilton equation. In this method tunneling rate is related to the change of Bekenstein Hawking entropy [36, 37]. 
In recent times, we have developed the Hawking radiation of Schwarzschild-de Sitter [40] and Schwarzschild- anti-de Sitter [41] black holes by Hamilton-Jacobi method when the selfgravitational interaction and the unfixed background spacetime are taken into account. In this method, the imaginary part of the action come from the relativistic Hamilton-Jacobi equation and the actual Hawking radiation spectrum deviates from the purely thermal one, satisfies the underlying unitary theory and gives a leading correction to the radiation spectrum. The imaginary part of the action for the process of s-wave emission across the horizon, which in turn is related to the change of Bekenstein Hawking entropy and using WKB approximation

$$
\Gamma \sim \exp (-2 \operatorname{Im} I)
$$

where $\Gamma$ is the emission rate, $I$ is the action for an outgoing positive energy particle.

Properties of black holes in anti-de Sitter (AdS) spaces especially those of thermodynamics [23] investigated thoroughly in recent years within the context of the conjectured equivalence [24, 47] and a large static black hole in asymptotically AdS spacetime corresponds to an (approximately) thermal state in the CFT. Therefore, the time scale for the decay of the black hole perturbation, which is given by the imaginary part of its action, corresponds to the timescale to reach thermal equilibrium in the strongly coupled CFT [7]. The accelerating expansion of our universe indicates the cosmological constant might be a positive one $[35,38,42]$ and the recent development in string /M-theory greatly stimulate the study of black holes in anti-de Sitter spaces and hence our study on the Kerr-anti-de Sitter black hole is plausible and meaningful.

In order to carry-over this article, KAdS black hole spacetime is described and near the event horizon the new line element of KAdS black hole is derived in the later section. In section 3, the unfixed background spacetime and the self-gravitational interaction are taken into account, we review the Hawking non-thermal radiation of KAdS black hole from massive particle tunneling method. In section 4, we derived the Hawking purely thermal radiation from non-thermal rate. Finally, in section 5, we present our remarks.

\section{Kerr-anti-de Sitter solution}

Kerr-anti-de Sitter black hole, which is the exact solution of the Einstein field equations with a negative cosmological constant describes rotating black hole in four-dimensional spacetime with asymptotic-anti-de Sitter behavior in the Boyer-Lindguist coordinates [11] with cosmological radius $l$, mass $M$ and the angular momentum per unit mass $a$ has the form

$$
\begin{aligned}
\mathrm{ds}^{2}=-\frac{1}{\rho^{2}}\left(\Delta_{\mathrm{r}}-\right. & \left.\Delta_{\theta} \mathrm{a}^{2} \sin ^{2} \theta\right) \mathrm{dt}^{2}+\frac{\rho^{2}}{\Delta_{\mathrm{r}}} \mathrm{dr} \mathrm{r}^{2}+\frac{\rho^{2}}{\Delta_{\theta}} \mathrm{d} \theta^{2}+\frac{1}{\rho^{2} \Xi^{2}}\left(\Delta_{\theta}\left(\mathrm{r}^{2}+\mathrm{a}^{2}\right)^{2}\right. \\
& \left.-\Delta_{\mathrm{r}} \mathrm{a}^{2} \sin ^{2} \theta\right) \sin ^{2} \theta \mathrm{d} \phi^{2}-\frac{2 a}{\rho^{2} \Xi}\left(\left(\mathrm{r}^{2}+\mathrm{a}^{2}\right) \Delta_{\theta}\right. \\
& \left.-\Delta_{\mathrm{r}} \sin ^{2} \theta\right) d t \mathrm{~d} \phi,
\end{aligned}
$$

where $\rho^{2}=r^{2}+a^{2} \cos ^{2} \theta, \Delta_{r}=\left(r^{2}+a^{2}\right)\left(1+\frac{r^{2}}{l^{2}}\right)-2 M r, \Delta_{\theta}=1-\frac{a^{2} \cos ^{2} \theta}{l^{2}}, \Xi=1-\frac{a^{2}}{l^{2}}$. 
The coordinates are defined such that $-\infty \leq t \leq \infty, r \geq 0,0 \leq \theta \leq \pi$ and $0 \leq \phi \leq 2 \pi$. The only positive real root is obtained from the zeroes of $r^{4}+\left(l^{2}+a^{2}\right)-2 M l^{2} r+l^{2} a^{2}=0$ and which is located at the black hole (event) horizon $r_{+}$such that

$r_{+}=\frac{l \alpha}{\sqrt{3}} \sinh \left[\frac{1}{3} \sinh ^{-1} \frac{3 \sqrt{3} M}{l \Xi \alpha}\right] \times\left(1+\sqrt{1-\frac{a^{2} l}{\sqrt{3} M \alpha} \cdot \frac{2}{1+\delta} \cdot \operatorname{cosech}\left[\frac{1}{3} \sinh ^{-1} \frac{3 \sqrt{3} M}{l \Xi \alpha}\right]}\right)$,

where $\delta=\sqrt{1-\frac{4 a^{2} \alpha^{2}}{3 M^{2}} \cdot \sinh ^{2}\left[\frac{1}{3} \sinh ^{-1} \frac{3 \sqrt{3} M}{l \Xi \alpha}\right]}, \alpha=\sqrt{1+\frac{a^{2}}{l^{2}}}$.

Expanding $r_{+}$in terms of Kerr-anti-de Sitter black hole parameters with $a^{2}\left(1-a^{2} / l^{2}\right)<1$ and setting $\delta=1$, we obtain

$$
r_{+}=\frac{1}{\Xi}\left(1-\frac{4 M^{2}}{l^{2} \Xi \alpha^{2}}+\ldots\right)\left(M+\sqrt{M^{2}-a^{2} \Xi}\right) .
$$

Obviously, the event horizon of the Kerr-anti-de-Sitter black hole is less than the Kerr event horizon $r_{K e}=M+\sqrt{M^{2}-a^{2}}$. It also gives Kerr black hole [56] for $\ell \rightarrow \infty(\Xi \rightarrow 1)$ and Schwarzschild-anti-de Sitter black hole [40] for $a=0$. To study the Hawking radiation of Kerranti-de Sitter black hole effectively, we choose dragging coordinate transformation as follows

$$
\frac{d \phi}{d t}=-\frac{g_{03}}{g_{33}}=\frac{a \Xi\left[\Delta_{\theta}\left(r^{2}+a^{2}\right)-\Delta_{r}\right]}{\Delta_{\theta}\left(r^{2}+a^{2}\right)^{2}-\Delta_{r} a^{2} \sin ^{2} \theta}
$$

Now applying (7) on the line element (2), then the new line element of the Kerr-anti-de Sitter black hole takes on form as

$$
d s^{2}=-\frac{\Delta_{r} \Delta_{\theta} \rho^{2}}{\Delta_{\theta}\left(r^{2}+a^{2}\right)^{2}-\Delta_{r} a^{2} \sin ^{2} \theta} d t^{2}+\frac{\rho^{2}}{\Delta_{r}} \mathrm{dr} r^{2}+\frac{\rho^{2}}{\Delta_{\theta}} \mathrm{d} \theta^{2} .
$$

The position of the event horizon is same as given in Eq. (6). We select the line element (8) and make a treatment to it and therefore, near the event horizon, the line element (8) can be written as

$d s^{2}=-\frac{\Delta_{r, r}\left(r_{+}\right)\left(r-r_{+}\right) \rho^{2}\left(r_{+}\right)}{\left(r_{+}^{2}+a^{2}\right)^{2}} d t^{2}+\frac{\rho^{2}\left(r_{+}\right)}{\Delta_{r, r}\left(r_{+}\right)\left(r-r_{+}\right)} \mathrm{dr}^{2}+\frac{\rho^{2}\left(r_{+}\right)}{\Delta_{\theta}} \mathrm{d} \theta^{2}$,

where $\rho^{2}\left(r_{+}\right)=r_{+}^{2}+a^{2} \cos ^{2} \theta$ and $\Delta_{\mathrm{r}, r}\left(r_{+}\right)=\frac{2}{\Xi^{2}}\left(\alpha^{2} r_{+}-M-2 \frac{r_{+}^{3}}{l^{2}}\right)$.

\section{The Hamilton-Jacobi Method for Non-thermal Radiation}

To calculate the imaginary part of the action from the relativistic Hamilton-Jacobi equation, we use the method of Chen et al. [10] by giving up the exploration of the equation of motion in the Painlev'e coordinates systems. In order to calculate the imaginary part of the action from the relativistic Hamilton-Jacobi equation, the action $I$ of the outgoing particle from the black hole horizon satisfies the relativistic Hamilton-Jacobi equation

$$
g^{a b}\left(\partial_{a} I\right)\left(\partial_{b} I\right)+m^{2}=0
$$

In which $m$ and $g^{a b}$ are the mass of the particle and the inverse metric tensors derived from the line element (9). The non-null inverse metric tensors for the metric (9) are 


$$
g^{00}=-\frac{\left(r_{+}^{2}+a^{2}\right)^{2}}{\Delta_{r, r}\left(r_{+}\right)\left(r-r_{+}\right) \rho^{2}\left(r_{+}\right)}, g^{11}=\frac{\Delta_{r, r}\left(r_{h}\right)\left(r-r_{+}\right)}{\rho^{2}\left(r_{+}\right)}, g^{22}=\frac{\Delta_{\theta}}{\rho^{2}\left(r_{+}\right)}
$$

Using (12) in Eq. (11), we have

$$
g^{00}\left(\partial_{t} I\right)^{2}+g^{11}\left(\partial_{r} I\right)^{2}+g^{22}\left(\partial_{\theta} I\right)^{2}+m^{2}=0 .
$$

The action $I$ for $I(t, r, \theta, \phi)$ is too difficult to solve. To find the solution in a convenient way, the separation of variables can be taken as follows

$$
I=-\omega t+R(r)+H(\theta)+j \phi,
$$

where $\omega$ is the energy of the particle, $j$ is the angular momentum with respect to the angular $\phi$, $R(r)$ and $H(\theta)$ are the generalized momentums.

From Eq.(13) and Eq. (14), we get

$$
\begin{aligned}
R(r) & = \pm \frac{r_{+}{ }^{2}+a^{2}}{\Delta_{r},{ }_{r}\left(r_{+}\right)} \int \frac{d r}{r-r_{r_{+}}} \times \sqrt{\left(\omega-j \Omega_{+}\right)^{2}-\frac{\rho^{2}\left(r_{r_{+}}\right) \Delta_{r},{ }_{r}\left(r_{r_{+}}\right)\left(r-r_{r_{+}}\right)}{\left(r_{r_{+}}{ }^{2}+a^{2}\right)^{2}}\left[\frac{\Delta_{\theta}}{\rho^{2}\left(r_{r_{+}}\right)} \cdot\left(\partial_{\theta} H(\theta)\right)^{2}+m^{2}\right]} \\
& = \pm \frac{\pi i\left(r_{+}{ }^{2}+a^{2}\right)}{\Delta_{r},{ }_{r}\left(r_{+}\right)}\left(\omega-j \Omega_{+}\right)+\eta
\end{aligned}
$$

where $\Omega_{+}=\frac{a \Xi}{\left(r_{+}^{2}+a^{2}\right)}=\frac{d \phi}{d t}$ at $r=r_{+}$express the angular velocity of the particle at the event horizon and \pm sign comes from the square root and $\eta$ is the constant of integration. Inserting Eq. (15) into Eq. (14), the imaginary part of two different actions corresponding to the outgoing and incoming particles can be written as

$$
\operatorname{Im} I_{ \pm}= \pm \frac{\pi\left(r_{+}{ }^{2}+a^{2}\right)}{\Delta_{r},_{r}\left(r_{+}\right)}\left(\omega-j \Omega_{+}\right)+\operatorname{Im}(\eta) .
$$

With classical limit as mentioned in refs. [33], we make sure the incoming probability to be unity when there is no reflection i.e., everything is absorbed by the horizon. In this situation the appropriate value of $\eta$ instead of zero or infinity can be taken as $\eta=\frac{\pi \mathrm{i}\left(\mathrm{r}_{+}^{2}+\mathrm{a}^{2}\right)}{\Delta_{r, r}\left(\mathrm{r}_{+}\right)}\left(\omega-\mathrm{j} \Omega_{+}\right)+$ $\operatorname{Re}(\eta)$. Therefore, $\operatorname{Im} I_{-}=0$ and $I_{+}$give the imaginary part of action $I$ corresponding to the outgoing particle of the form

$$
\begin{aligned}
\operatorname{Im} I & =\frac{2 \pi\left(r_{+}{ }^{2}+a^{2}\right)}{\Delta_{r},_{r}\left(r_{+}\right)}\left(\omega-j \Omega_{+}\right) \\
& =\frac{\Xi^{2} \pi\left(r_{+}^{2}+a^{2}\right)}{\alpha^{2} r_{+h}-M-2 \frac{r_{+}^{3}}{l^{2}}}\left(\omega-j \Omega_{+}\right) \\
& =\frac{\Xi^{2} \pi r_{+}^{2}}{\alpha^{2} r_{+h}-M-2 \frac{r_{+}^{3}}{l^{2}}} \omega+\frac{\Xi^{2} \pi a^{2}}{\alpha^{2} r_{+h}-M-2 \frac{r_{+}^{3}}{l^{2}}} \omega-\frac{\Xi^{3} \pi a}{\alpha^{2} r_{+h}-M-2 \frac{r_{+}^{3}}{l^{2}}} j
\end{aligned}
$$


Using Eq. (6) into Eq. (17), we get the imaginary part of the action as follows-

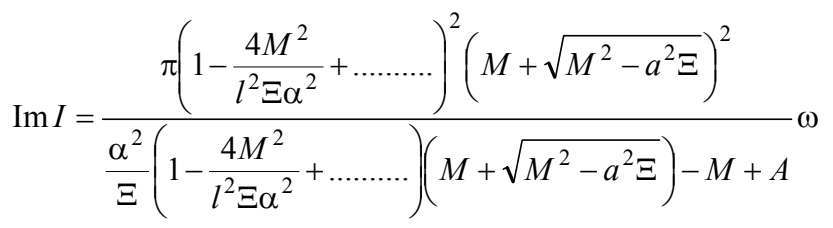

$$
\begin{aligned}
& +\frac{\Xi^{2} \pi a^{2}}{\frac{\alpha^{2}}{\Xi}\left(1-\frac{4 M^{2}}{l^{2} \Xi \alpha^{2}}+\ldots \ldots \ldots .\right)\left(M+\sqrt{M^{2}-a^{2} \Xi}\right)-M} \omega-\frac{\Xi^{3} \pi a}{\frac{\alpha^{2}}{\Xi}\left(1-\frac{4 M^{2}}{l^{2} \sum \alpha^{2}}+\ldots \ldots \ldots . .\right)\left(M+\sqrt{M^{2}-a^{2} \Xi}\right)-M+A} j,
\end{aligned}
$$

where $A==\frac{2}{l^{2} \Xi^{3}}\left(1-\frac{4 M^{2}}{l^{2} \Xi \alpha^{2}}+\ldots \ldots \ldots . . .\right)^{3}\left(M+\sqrt{M^{2}-a^{2} \Xi}\right)^{3}$

For the simplicity, after some calculations, neglecting $M^{3}$ and its higher order terms, we then get

$$
\operatorname{Im} I=\frac{\pi \Xi}{\alpha^{2}} \cdot \frac{\left(M+\sqrt{M^{2}-a^{2} \Xi}\right)^{2}}{\left(M+\sqrt{M^{2}-a^{2} \Xi}\right)-\frac{M \Xi}{\alpha^{2}}} \omega+\frac{\Xi^{3} \pi a^{2}}{\alpha^{2}\left(M+\sqrt{M^{2}-a^{2} \Xi}\right)-\frac{M \Xi}{\alpha^{2}}} \omega-\frac{\Xi^{4} \pi a}{\alpha^{2}\left(M+\sqrt{M^{2}-a^{2} \Xi}\right)-\frac{M \Xi}{a^{2}}} j
$$

Suppose the Arnowitt-Deser-Misner (ADM) mass of the total spacetime to be fixed and in presence of cosmological constant KAdS spacetime is dynamic, and hence allow KAdS black hole to fluctuate. When a particle with energy $\omega$ and angular momentum $j$ tunnels out, the mass and angular momentum of the KAdS black hole should be replaced by $M-\omega$ and $J-j$ respectively. Taking the self-gravitational interaction into account, the imaginary part of the true action can be calculated from Eq. (19) in the following integral form

$$
\begin{aligned}
\operatorname{Im} I= & \frac{\pi \Xi}{\alpha^{2}} \cdot \int_{0}^{\omega} \frac{\left(M+\sqrt{M^{2}-a^{2} \Xi}\right)^{2}}{\sqrt{M^{2}-a^{2} \Sigma}+\left(M-\frac{M \Xi}{\alpha^{2}}\right)} d \omega^{\prime}+\frac{\pi \Xi^{3}}{\alpha^{2}} \int_{0}^{\omega} \frac{a^{2}}{\sqrt{M^{2}-a^{2} \sum}+\left(M-\frac{M \Xi}{\alpha^{2}}\right)} d \omega^{\prime}- \\
& \frac{\pi \Xi^{4}}{\alpha^{2}} \int_{0}^{j} \frac{a}{\sqrt{M^{2}-a^{2} \Sigma}+\left(M-\frac{M \Xi}{\alpha^{2}}\right)} d j^{\prime}
\end{aligned}
$$

For the maximum value of integration, neglecting $\left(1-\frac{\Xi}{\beta^{2}}\right) M$ and, then replacing $M$ by $M-\omega$ and $j$ by $J-j$, and then using $J-j=\left(M-\omega^{\prime}\right) a / \Sigma^{2}$, equation (20) becomes

$$
\operatorname{Im} I=-\frac{\pi \Xi}{a^{2}} \cdot \int_{M}^{M-\omega} \frac{\left(M-\omega+\sqrt{(M-\omega)^{2}-a^{2} \Xi}\right)^{2}}{\sqrt{(M-\omega)^{2}-a^{2} \Xi}} d\left(M-\omega^{\prime}\right)
$$




$$
=-\frac{\pi \Xi}{\alpha^{2}} \cdot \int_{M}^{M-\omega} \frac{2(M-\omega)^{2}+2(M-\omega) \sqrt{(M-\omega)^{2}-a^{2} \Xi}}{\sqrt{(M-\omega)^{2}-a^{2} \Xi}} d\left(M-\omega^{\prime}\right)+\frac{\pi \Xi}{\alpha^{2}} \cdot \int_{M}^{M-\omega} \frac{a^{2} \Xi}{\sqrt{(M-\omega)^{2}-a^{2} \Xi}} d\left(M-\omega^{\prime}\right)
$$

Finishing the $\omega^{\prime}$ integral finally yields

$$
\begin{aligned}
\operatorname{Im} I & =-\frac{\pi \Xi}{a^{2}}\left\{(M-\omega) \sqrt{(M-\omega)^{2}-a^{2} \Xi}+(M-\omega)^{2}-M \sqrt{M^{2}-a^{2} \Xi}-M^{2}\right\} \\
& =-\frac{\pi \Xi}{2 \alpha^{2}}\left\{2(M-\omega) \sqrt{(M-\omega)^{2}-a^{2} \Xi}+2(M-\omega)^{2}-2 M \sqrt{M^{2}-a^{2} \Xi}-2 M^{2}\right\} \\
& =-\frac{1}{2} \exp \left[\pi\left(r_{f}{ }^{2}-r_{i}{ }^{2}\right)\right]=-\frac{1}{2} \exp \left(\Delta S_{B H}\right),
\end{aligned}
$$

where $\Delta S_{B H}=S_{B H}(M-\omega)-S_{B H}(M)$ is the change of Bekenstein-Hawking entropy of the the Kerr-anti-de Sitter black hole before and after the emission of particles by setting $r_{i}=$ $\frac{\sqrt{\Xi}}{\alpha}\left[M+\sqrt{M^{2}-a^{2} \Xi}\right]$ and $r_{f}=\frac{\sqrt{\Xi}}{\alpha}\left[(M-\omega)+\sqrt{(M-\omega)^{2}-a^{2} \Xi}\right]$ respectively.

Therefore, using Eq. (1) the tunneling probability for the KAdS black hole can be obtained as

$\Gamma \sim \exp (-2 \operatorname{Im} I)=\exp \left(\Delta S_{B H}\right)$

The result shows the actual radiation spectrum deviates from the purely thermal one and gives a correction to the Hawking radiation of the black hole.

\section{Purely Thermal Radiation}

It is obvious from Eq. (23) the radiation spectrum is not pure thermal although gives a correction to the Hawking radiation of KAdS black hole. The purely thermal spectrum can be derived from Eq. (23) by expanding as discussed by Hossain et al. [40, 41] of the form

$$
\Gamma \sim \exp \left(\Delta S_{B H}\right)=\exp \left\{-\omega \frac{\partial S_{B H}(M)}{\partial M}+\frac{\omega^{2}}{2} \frac{\partial^{2} S_{B H}(M)}{\partial M^{2}}\right\}
$$

From Eq. (22), we can write

$$
S_{B H}(M-\omega)=\frac{\pi \Xi}{\alpha^{2}}\left\{(M-\omega)+\sqrt{(M-\omega)^{2}-a^{2} \Xi}\right\}^{2}
$$

From above two equations, we obtain the purely thermal emission rate of the form

$$
\Gamma \sim \exp \left(\Delta S_{B H}\right)=\exp \left[\begin{array}{c}
-\frac{2 \pi \Xi \omega}{\alpha^{2}}\left\{\left(2 M+\sqrt{M^{2}-a^{2} \Xi^{2}}+\frac{M^{2}}{\sqrt{M^{2}-a^{2} \Xi^{2}}}\right)\right. \\
\left.-\frac{\omega}{2}\left(2+\frac{3 M}{\sqrt{M^{2}-a^{2} \Xi^{2}}}-\frac{M^{3}}{\sqrt{M^{2}-a^{2} \Xi^{2}}}\right)\right\}
\end{array}\right]
$$

In the limiting case i.e. when $\Lambda=0$, then $\Xi=1, \alpha=1$ and in this case, non-thermal and purely thermal tunneling rates for the KAdS black hole reduces to 
$\Gamma \sim \exp (-2 \operatorname{Im} I)=\exp \left\{\pi\left[\left((M-\omega)+\sqrt{(M-\omega)^{2}-a^{2}}\right)^{2}-\left(M+\sqrt{M^{2}-a^{2}}\right)^{2}\right]\right\}$

and

$\Gamma \sim \exp \left(\Delta S_{B H}\right)=\exp \left[-2 \pi \omega\left\{\left(2 M+\sqrt{M^{2}-a^{2}}+\frac{M^{2}}{\sqrt{M^{2}-a^{2}}}\right)-\frac{\omega}{2}\left(2+\frac{3 M}{\sqrt{M^{2}-a^{2}}}-\frac{M^{3}}{\sqrt{M^{2}-a^{2}}}\right)\right\}\right]$.

Which are consistent to the results for the Kerr black hole [56] and where $r_{i}=M+\sqrt{M^{2}-a^{2}}$ and $r_{f}=(M-\omega)+\sqrt{(M-\omega)^{2}-a^{2}}$ and $r_{i}=M+\sqrt{M^{2}-a^{2}}$. These are the positions of the event horizon of Kerr black hole before and after the emission of the particles respectively.

For another special case, only when $a=0$, the line element (2) is reduced to the SAdS black hole [40]. So the tunneling probabilities for the SAdS black hole can be written as

$$
\Gamma \sim \exp \left(\Delta S_{B H}\right)=\exp \left\{\pi\left[4(M-\omega)^{2}\left(1-\frac{8(M-\omega)^{2}}{l^{2}}\right)-4 M^{2}\left(1-\frac{4 M^{2}}{l^{2}}\right)\right]\right\}
$$

and $\Gamma \sim \exp \left(\Delta S_{B H}\right)=\exp \left\{-8 \pi \omega\left[4\left(M-\frac{16 M^{3}}{l^{2}}\right)-\frac{\omega}{2}\left(1-\frac{48 M^{2}}{l^{2}}\right)\right]\right\}$,

where $r_{i}=2 M\left(1-\frac{4 M^{2}}{l^{2}}\right)$ and $r_{f}=2(M-\omega)\left(1-\frac{4(M-\omega)^{2}}{l^{2}}\right)$ are the locations of the SAdS [40] event horizon before and after the particles emission.

Furthermore only when $a=0$ and $\Lambda=0$, the line element (2) is reduced to the Schwarzschild black hole [36] and therefore the non-thermal and purely thermal tunneling rates can be written as

$$
\begin{aligned}
& \Gamma \sim \exp (-2 \operatorname{Im} I)=\exp \left\{\pi\left[4(M-\omega)^{2}-4 M^{2}\right)\right]=\exp \left[\pi\left(r_{f}^{2}-r_{i}^{2}\right)\right] \text { and } \\
& \Gamma \sim \exp \left(\Delta S_{B H}\right)=\exp \left\{-8 \pi \omega\left(M-\frac{\omega}{2}\right)\right\}
\end{aligned}
$$

which are full accordant with Parikh and Wilczek's results [36] and where $r_{i}=2 M$ and $r_{f}=2(M-$ $\omega)$ are the locations of the Schwarzschild black hole event horizon before and after the particles emission.

\section{Concluding Remarks}

We have developed Hawking radiation as massive particle tunneling method from KAdS black hole in this paper. Considering the self-gravitational interaction, the background spacetime as dynamical and the energy as conservation, we have explored that the non-thermal and purely thermal tunneling probabilities of KAdS black hole at the event horizon. The non-thermal tunneling probability depending on the initial and final entropy of the system is called the change Bekenstein-Hawking entropy and it is noted that the similar results have been shown considering the same proposition for massive particle tunneling at the event horizon of SAdS [41] and TaubNUT [9] black holes and also agree by massless or massless charged particle tunneling from various spacetime like as charged black hole with a global monopole [18], kerr-NUT black hole [27], Kerr and Kerr-Newmann black holes [17] etc. Thus the actual radiation spectrum of KAdS 
black hole is not precisely thermal and the tunneling probability is related to the change of Bekenstein-Hawking entropy but satisfies an underlying unitary theory and also gives a correction to Hawking radiation.

\section{REFERENCES}

[1] Ali M. H., Hawking radiation via tunneling from hot NUT-KerrNewman-Kasuya spacetime, Class. Quant. Grav 24 (2007), 5849-5860.

[2] Arzano M., M.Medved A.J.M. Vagenas and Vagenas E. C., Hawking Radiation as tunnelling through the Quantum Horizon, JHEP 0509, 037 (2005), 27599-3255.

[3] Angheben M., Nadalini M., Vanzo L. and Zerbini S., Hawking radiation as tunneling for extremal and rotating black holes, JHEP 05 (2005), 014.

[4] Bardeen J. M., Carter B. and Hawking S. W., The four laws of black hole mechanics, Math. Phys. 31 (1973), 161-170.

[5] Bekenstein, J.D., Generalised second law of thermodynamics in black hole physics, Phys. Rev. D 9 (1974), 3292.

[6] Bekenstein J. D., Black Holes and Entropy, Phys. Rev. D 7(8) (1973), 2333-2346.

[7] Birmingham D., Sachs I. and Solodukhin S.N., Relaxation in conformal field theory, Hawking-Page transition, and quasinormal or normal modes, Phys. Rev. D 67 (2003), 104026.

[8] Chen D. Y., Yang H. T., Zu X. T., Fermion tunneling from anti-de Sitter spaces, Eur. Phys. J. C 56 (2008), 119-124.

[9] Chen D. Y., Jiang Q. Q., Zu X. T., Hawking radiation of Dirac particles via tunnelling from rotating black holes in de Sitter spaces, Phys. Lett. B 665 (2008), 106-110 ; Chen D. Y., Jiang Q. Q., Zu X. T., Fermions tunneling from the charged dilatonic black holes, Class. Quant. Grav. 25 (2008), 205022.

[10] Chen D. Y., Zu X. T. and Yang S. H., Massive particle Tunnels From The Taub-Nut Black Hole, Acta Physica Polonica B 39(6), (2008), 1329-1336.

[11] Carter B., The commutation property of a stationary, axisymmetric system, Commun. Math. Phys. 17 (1979), 233-238.

[12] Hawking S. W., Black hole explosions, Nature (London) 248 (1974), 30-31.

[13] Hawking S. W., Particle Creation By Black Holes, Commun. Math. Phys. 43 (1975), 199-220.

[14] Hemming S. and Keski-Vakkuri E., Hawking radiation from AdS black holes, Phys. Rev. D 64 (2001), 044006.

[15] Hartle J. B. and Hawking S. W., Path-integral derivation of black-hole radiance, Phys. Rev.D 13 (1976), 2188-2203.

[16] Jiang K. X., Ke S. M.and Peng D.T., Hawking radiation as tunneling and the unified first law of thermodynamics for a class of dynamical black holes, Int. J. Mod. Phys. D 18 (2009), 1707-1717

[17] Jiang, Q.Q., Wu, S.Q.and Cai, X. : Hawking radiation as tunneling from the Kerr and Kerr-Newman black holes, Phys. Rev. D 76 (2006), 064003.

[18] Jiang Q. Q., Wu S. Q., Hawking radiation of charged particles as tunnelling from Reissner-Nordstromde Sitter black holes with a global monopole, Phys. Lett. B 635 (2006), 151-155.

[19] Kraus P., Wilezek F., Self-Interaction Correction to Black Hole Radiance, Nucl. Phys. B 433 (1995), 403-420 ; Effect of Self-Interaction on Charged Black Hole Radiance, Nucl. Phys. B 437 (1995), 231242.

[20] Kraus P. and Wilczek F., Effect of Self-Interaction on Charged Black Hole Radiance, Nucl. Phys. B 437 (1995), 231-242.

[21] Kim S. P., Hawking Radiation as Quantum Tunneling in Rindler Coordinate, JHEP 11 (2007), 048. 
[22] Kim S. P., Schwinger Mechanism and Hawking Radiation as Quantum Tunneling, J. Korean Phys. Soc. 53 (2008), 1095-1099.

[23] Kerner R. and Mann R. B., Tunnelling, Temperature, and Taub-NUT Black Holes, Phys. Rev. D 73 (2006), 104010; Tunnelling from Godel black holes, Phys. Rev. D 75 (2007), 084022.

[24] Klemen D., Some aspects of the de Sitter/CFT correspondence, Nucl. Phys. B 625 (2002), 295-311.

[25] Liu C. Z., Zhang J. Y. and Zhao Z., Charged particle's tunneling from a dilaton black hole, Phys. Lett. $B 639$ (2006), 670-674.

[26] Liu W. B., New coordinates for BTZ black hole and Hawking adiation via tunneling, Phys. Lett. B 634 (2006), 541-544.

[27] Liu H. L., Liu Z. X., Hou J. S., Yang S. Z., A New Method to Study the Hawking Radiation from the Kerr-NUT Black Hole, Int. J. Theor. Phys.47 (2008), 2960-2965.

[28] Medved A.J.M., Radiation via tunnelling from a de Sitter cosmological horizon, Phys. Rev. D 66 (2002), 124009.

[29] Matsuno K. and Umetsu K., Hawking radiation as tunneling from squashed Kaluza-Klein black hole, Phys. Rev. D 83 (2011), 064016.

[30] Medved A. J. M., Radiation via tunneling in the charged BTZ black hole, Class. Quantum Grav. 19 (2002), 589-598.

[31] Medved A. J.M. and Vagenas E.C., On Hawking radiation as tunneling with logarithmic corrections, Mod. Phys. Lett. A 20 (2005), 2449-2454.

[32] Mehdipour S. H., Hawking radiation as tunneling from a Vaidya black hole in noncommutative gravity, Phys. Rev. D 81 (2010), 124049.

[33] Mitra P., Hawking temperature from tunnelling formalism, Phys.Lett. B 648 (2007), 240-242.

[34] Nozari K. and Mehdipour S. H., Hawking radiation as quantum tunneling from a noncommutative Schwarzschild black hole, Class. Quant. Grav. 25 (2008), 175015.

[35] Ostriker J.P. and Steinhardt P.J., The observational case for a low-density Universe with a non-zero cosmological constant, Nature 377 (1995), 600-602.

[36] Parikh M. K and Wilczek F., Hawking Radiation as Tunneling, Phys. Rev. Lett. 85 (2000), 5042-5045.

[37] Parikh M. K., New Coordinates for de Sitter Space and de Sitter Radiation, Phys. Lett. B 546 (2002), 189-195.

[38] Perlmutter S. et al., Measurements of $\Omega$ and $\Lambda$ from 42 High Redshift Supernovae, Astrophys. $J$. 517(2): (1999), 565-586.

[39] Robinson S. P., Wilczek F., Relationship between Hawking Radiation and Gravitational Anomalies, Phys. Rev. Lett. 95 (2005), 011303 [gr-qc/0502074].

[40] Rahman M. A., Hossain M. I., Hawking radiation of Schwarzschild-de Sitter black hole by HamiltonJacobi method, Phys. Lett. B 712 (2012), 1-5.

[41] Rahman M. A., Hossain M. I., Hawking non-thermal and thermal radiations of Schwarzschild anti-de Sitter black hole by Hamilton-Jacobi method, Astrophys Space Sci. vol. 345 (2013), 345-350.

[42] Reiss A. G. et al., Observational evidence from supernovae for an accelerating universe and a cosmological constant, Astron. J. 116 (1998), 1009-1038.

[43] Sarkar S. and Kothawala D., Hawking radiation as tunneling for spherically symmetric black holes: A generalized treatment, Phys. Lett. B 659 (2008), 683-687.

[44] Shankaranarayanan S., Srinivasan K., Padmanabhan T., Method of complex paths and general covariance of Hawking radiation, Mod. Phys. Lett. A 16 (2001), 571-578.

[45] Shankaranarayanan S., Srinivasan K., Padmanabhan T., Hawking radiation in different coordinate settings: Complex paths approach, Class Quant. Grav. 19 (2002), 2671-2688.

[46] Srinivasan K. and Padmanabhan T., Particle production and complex path analysis, Phys. Rev. D 60 (1999), 024007(1-20). 
[47] Strominger A., The dS/CFT correspondence, JHEP 010 (2001), 034; Klemm D., Nucl. Phys. B 625 (2002), 296-306.

[48] Usman A., Gillani K., Saifullah K., Tunneling of Dirac particles from accelerating and rotating black holes, Phys. Lett. B 699 (2011), 15-20.

[49] Vagenas E. C., Two-dimensional dilatonic black holes and Hawking radiation, Mod. Phys. Lett. A 17 (2002), 609-618.

[50] Vagenas E.C., Semiclassical corrections to the Bekenstein-Hawking entropy of the BTZ black hole via self-gravitation, Phys. Lett. B $\mathbf{5 3 3}$ (2002), 302-306.

[51] Vagenas E.C., Generalization of the KKW analysis for black hole radiation, Phys. Lett.B 559 (2003), 65-73.

[52] Wu S. Q., Jiang Q. Q., Remarks on Hawking radiation as tunneling from the BTZ black holes, JHEP 03 (2006), 079.

[53] Wu X. N., Gao S., Tunneling effect near a weakly isolated horizon, Phys. Rev. D. 75 (2007), 044027.

[54] Yang S.Z., Kerr-Newman-Kasuya black hole tunnelling radiation, Chin. Phys. Lett. 22(10) (2005), 2492-2495; Yang S. Z., Jiang Q. Q. and Li H. L., Quantum tunnelling radiation of Einstein-MaxwellDilaton Axion black hole, Chin. Phys. 14(12) (2005), 2411-04.

[55] Zhang J. Y., Zhao Z., New coordinates for Kerr-Newman black hole radiation, Phys. Lett. B 618 (2005), 14-22.

[56] Zhang J.Y. and Zhao Z., Hawking Radiation Via Tunneling From Kerr Black Holes, Mod. Phys. Lett. A 20, 22 (2005), 1673-1681.

[57] Zhang J. Y. and Zhao Z., Hawking radiation of charged particles via tunnelling from the ReissnerNordstrom black hole, JHEP 10 (2005), 055.

[58] Zhang J. Y., Zhao Z., Massive particles' black hole tunneling and de Sitter tunneling, Nucl. Phys. B 725 (2005), 173-180.

[59] Zhang, J.Y. and Zhao Z., Charged particles' tunnelling from the Kerr-Newman black hole, Phys. Lett. $B 638$ (2006), 110-113. 